\title{
The role of socio-economic position as a moderator of children's healthy food intake
}

\author{
Dorota M. Zarnowiecki ${ }^{1 *} \dagger$, Natalie Parletta ${ }^{2} \dagger$ and James Dollman ${ }^{1} \dagger$ \\ ${ }^{1}$ Sanson Institute for Health Research, School of Population Health, University of South Australia, City East Campus, \\ Frome Road, GPO Box 2471, Adelaide, SA 5001, Australia \\ ${ }^{2}$ Exercise for Health and Human Performance Research Group, School of Health Sciences, University of South Australia, \\ City East Campus, Frome Road, GPO Box 2471, Adelaide, SA 5001, Australia
}

(Submitted 13 November 2013 - Final revision received 2 April 2014-Accepted 6 May 2014-First published online 23 June 2014)

\begin{abstract}
Children of low socio-economic position (SEP) consume poorer diets than those of high SEP; however, there is limited understanding of why socio-economic gradients in diet occur. Some evidence suggests that determinants of dietary intake may differ between SEP groups. The aim of the present study was to determine whether the associations between personal and environmental variables and children's fruit and vegetable intake, and healthy dietary behaviours are moderated by SEP. A total of 395 children aged 9 to 13 years and their parents were recruited in Adelaide, South Australia. Personal and environmental dietary predictors were measured using child-completed online questionnaires and telephone interviews with parents. Dietary intake was measured using an online FFQ. First, dietary predictors were identified using correlated component regression, and subsequently tested for moderation by four SEP indicators using partial least-squares structural equation modelling. Fruit and vegetable intake and healthy behaviours were predicted by self-efficacy, attitudes and a supportive home environment. For girls, only the associations of self-efficacy with healthy behaviours were moderated by occupation. For boys, income moderated the associations of fruit and vegetable intake with attitudes, and healthy behaviours with supportive home environments. Occupation and employment moderated the associations of boys' family environments and fruit intake, and attitudes with healthy behaviours. Reducing socio-economic disparities in children's healthy dietary intake may be more successfully achieved by tailoring health promotion policies and interventions according to variables that moderate the relationships between dietary intake and SEP.
\end{abstract}

Key words: Socio-economic position: Children: Healthy food intake: Fruit and vegetables: Moderation

Research into socio-economic gradients in health has indicated that children of low socio-economic position (SEP) are more likely to be overweight and obese, engage in less physical activity, spend more time in sedentary activities and have poorer quality of dietary intake than children of high $\mathrm{SEP}^{(1-3)}$. SEP has been identified as one of the strongest, most consistent correlates of children's fruit and vegetable intake ${ }^{(4)}$, and some evidence suggests that the gap between low- and high-SEP children with respect to diet quality may be widening. Between 2001 and 2008, consumption of fruit and vegetables among 10- to 12-year-old Norwegian children of low SEP fell from 13.9 to 12.6 eating occasions per week, whereas consumption among high-SEP children increased slightly from $14 \cdot 8$ to $15 \cdot 0$ eating occasions per week ${ }^{(5)}$. Given that children's dietary intake appears to be poor overall $^{(6)}$, and children of low SEP may have poorer dietary intakes than other children, this group forms an important target for improving dietary intake. However, there is currently limited understanding of socio-economic gradients in children's dietary intake, and how to target interventions to reduce these disparities.

Socio-economic gradients in children's diet may be explained in part by the differences between socio-economic groups in relation to personal and environmental determinants of children's dietary intake ${ }^{(5,7-10)}$. Children and adolescents of high SEP may have stronger preferences for fruits and vegetables, knowledge of dietary guidelines, and intentions to meet those guidelines ${ }^{(8-11)}$. Cognitive factors such as nutrition knowledge, self-efficacy for healthy eating and attitudes may act as mediators for explaining the associations of SEP

Abbreviations: CATI, computer-assisted telephone interview; CCR, correlated component regression; CNQ, Child Nutrition Questionnaire; SEP, socio-economic position.

*Corresponding author: D. M. Zarnowiecki, fax +61 8302 2794, email dorota.zarnowiecki@unisa.edu.au

t These authors contributed equally to this work. 
with children's dietary intake ${ }^{(7,12,13)}$, providing a mechanism through which SEP influences children's dietary choices. Adolescents of low SEP may eat less fruits and vegetables due to lower access at home ${ }^{(7,8,14)}$ and receive less support for healthy eating from their family, which may mediate SEP differences in fruit and vegetable intake ${ }^{(7,15)}$.

Apart from such mediating explanations, determinants of children's eating behaviours may differ between socioeconomic groups; that is, SEP may act as a moderator. Moderating variables can affect the strength and direction of the relationships between predictors and outcome variables, as distinct from mediating variables that provide a mechanism through which a predictor influences the outcome variable $^{(16,17)}$. Few studies have considered how predictordietary intake relationships may be moderated by SEP in children aged 9 to 13 years, with mixed findings overall. For example, two studies have found no moderator effects of a composite SEP measure and parents' education on the predictors of children's and young adolescents' fruit and vegetable intake ${ }^{(18,19)}$. In contrast, the snack intake of low-educated but not high-educated 15-year-old adolescents was positively related to the snack intake of others in their friendship group $^{(10)}$. In low-affluence but not high-affluence families of Hong Kong adolescents, lower perceived availability of fruits and vegetables in restaurants was associated with lower intake ${ }^{(9)}$. Sandvik et $a l^{(15)}$ found that in relation to 11 - to 12-year-old children's fruit intake, home fruit availability was significantly related to fruit intake in high-SEP children and not in middle- and low-SEP children, whereas self-efficacy was most strongly associated with intentions to eat fruit in low-SEP children.

These studies provide some indication that SEP may moderate the predictors of children's dietary intake. However, across these studies, a number of different SEP indicators were tested as moderators, across a broad range of predictor-dietary intake relationships. Given this, it is difficult to compare studies to gain a robust picture of socioeconomic disparities in the predictors of children's dietary intake. A better understanding is needed of how the drivers of dietary intake differ between socio-economic groups, in order to identify whether it is more efficacious to tailor health promotion strategies and interventions to the unique needs of socio-economic groups. The aim of the present study was to explore the interactions of personal and environmental predictors of children's fruit and vegetable intake, and healthy behaviours with SEP. The study adds to the existing literature by first using a social-ecological framework to identify both personal and environmental predictors of children's healthy food intake, and, second, by testing identified predictor-dietary intake relationships for moderation by four SEP indicators that have been found to be consistently associated with children's dietary intake. The findings contribute to our understanding of the benefits of tailoring health promotion for different socio-economic groups, and provide direction for which intervention components could be emphasised in the development of tailored interventions.

\section{Methods}

\section{Participants and recruitment}

The present study was a cross-sectional study of personal and environmental predictors of 9- to 13-year-old children's dietary intake. Data collection was conducted in Adelaide, South Australia from February to November 2010, and involved two phases: in phase 1, children completed the Child Nutrition Questionnaire (CNQ), and in phase 2, parents completed computer-assisted telephone interviews (CATI). The present study was conducted according to the guidelines laid down in the Declaration of Helsinki, and all procedures involving human subjects/patients were approved by the University of South Australia's Human Research Ethics Committee and the South Australian Department of Education and Children's Services Ethics Committee. Written informed consent was received from parents, and children provided verbal consent to participate in the study at the beginning of the first session.

Participants were recruited from grade 5 to 7 classes of primary schools in the Adelaide metropolitan area. Schools were stratified into tertiles of SEP using the school card register, a school ranking representing the proportion of students receiving means-tested government assistance to meet the cost of school attendance. Schools were randomly selected from each tertile, and study information was distributed to the principal of each selected school by email. A total of eightytwo schools were approached with information about the study, and twenty-seven schools participated in the study. The overall school response rate was $32 \cdot 1 \%$; however, this rate varied across SEP from $27 \cdot 8 \%$ for low-SEP, $30.0 \%$ for middle-SEP to $42.3 \%$ for high-SEP schools. All children and parents who were able to communicate in English with sufficient fluency to complete questionnaires and interviews were eligible to participate. In total, 2575 children received study information, and 1257 parents consented to participate (48.8\% response rate), of which 1201 children were present at school on the day of testing and participated in phase 1 . The response rate was lowest in low-SEP participants (43.1\%) and increased across SEP to $55.6 \%$ in high-SEP participants. Of the participating children, 1059 completed the $\mathrm{CNQ}$ and were eligible for their parents to complete the CATI in phase 2. The required sample size for the present study was determined to be 400 participants using multivariate regression analysis with fifteen to twenty variables and $\alpha=0.05$ powered at 0.08 . To achieve the required sample size, 525 parents were randomly selected and contacted by telephone to participate in the CATI, of which 400 completed the interview $(76.2 \%$ response rate). Of the children who completed the questionnaire, five were excluded as their responses to the CNQ were found to be incomplete, providing a total of 395 sets of matched data from children and parents for the present analysis.

\section{Protocol}

Phase 1 was conducted at schools during data collection sessions of approximately $90 \mathrm{~min}$ duration. Children completed online questionnaires using school computer facilities 
in groups ranging from ten to thirty students, guided by three to four research assistants. More research assistants were allocated to schools where literacy levels were likely to be low to provide adequate support for completing the questionnaires. Phase 2 CATI were designed and compiled by study researchers, and conducted at the completion of phase 1 data collection. The CATI lasted for $15-20 \mathrm{~min}$, and it was requested that the parent responsible for food provision complete the interview.

\section{Study measures}

The CNQ was adapted from an existing nutrition questionnaire developed and validated by Wilson et al. ${ }^{(20)}$ in young children of similar age range to that reported in the present study. Section 1 comprised a semi-quantitative FFQ from which three scores of children's usual intake of fruits and vegetables and healthy eating behaviours were derived ${ }^{(20)}$. Healthy eating behaviours comprised five behaviours that can contribute to healthy dietary intake, including (1) eating breakfast, (2) carrying a water bottle, (3) helping with groceries, (4) helping prepare dinner and (5) eating dinner with the family. To capture the usual patterns of consumption, children reported food intake for the day the CNQ was administered, the previous day and 'usual' intake. Dietary intake scores were derived by summing the responses to items forming each scale, as shown in more detail in Table 1. If one or more items within a score were missing, that score was not calculated. The original scale measuring children's engagement in eight 'healthy behaviours' was adapted into two scales with improved internal consistency, representing 'healthy behaviours' (Cronbach's $\alpha=0.54$ ) and 'unhealthy behaviours' (Cronbach's $\alpha=0.57$ ). All other scales used have been described in detail by Wilson et al. ${ }^{(20)}$. The dietary intake scores have been validated against $7 \mathrm{~d}$ food diaries and shown to be valid and reliable ${ }^{(20)}$.

According to the social-ecological framework used in the present study, a combination of personal and environmental factors influence children's eating behaviours, and a symbiotic relationship exists between these factors whereby the environment may facilitate an individual's engagement in eating behaviours, while personal attributes determine an individual's response to environmental factors ${ }^{(21,22)}$. In the present study, twenty-six personal and environmental predictors of children's eating behaviours were measured, of which eight were formed from children's responses to the CNQ, and eighteen were derived from parents' CATI responses (Table 1). Where possible, scores were utilised from existing validated instruments ${ }^{(20,23-26)}$, while some scores were developed for the present study using confirmatory factor analysis to form scales with appropriate properties (data not presented here, but available upon request). Cronbach's $\alpha$ values ranged from 0.52 to 0.90 , indicating acceptable internal consistency as determined by an $\alpha$ value of at least 0.70 , or 0.50 for scores with less than ten items ${ }^{(27)}$. Supportive and unsupportive home environment scales were formed from children's responses to items about parents' encouragement of healthy food intake, home food availability and accessibility, and parent modelling. Additionally, parents reported home food availability in two separate scales (Table 1). The parent upbringing in relation to food scale included two items on whether parents were taught about healthy eating and how to cook as children. The neighbourhood food environment scale measured the availability and quality of fruit and vegetables in the neighbourhood.

\section{Socio-economic variables}

Parents reported demographic information about their families in the CATI (Table 2). Maternal education, occupation, employment and household income were tested as moderating SEP variables. Mothers' education was used as it has been shown to be consistently associated with children's food intake $e^{(4)}$, and most mothers still have primary responsibilities in the household for feeding children ${ }^{(28)}$. Education level was reported on an 8-point scale ranging from (1) never attended school to (8) completed postgraduate education. Occupation and employment have also been reported to be consistently associated with predictors of children's dietary intake, and some evidence suggests that they moderate predictor-dietary intake associations ${ }^{(15)}$. Mother's job title was coded using the eight-tier Australian and New Zealand Standard Classification of Occupations that hierarchically groups occupations requiring similar levels of skills, education, responsibility and experience ${ }^{(29)}$. An additional category was created for individuals 'not in the labour force', comprising individuals engaged in full-time home duties, retired persons, unemployed and students. Where insufficient information was provided in the job title to accurately classify an individual, for example 'public servant', these data points were not coded and were treated as missing data. An additional variable representing maternal employment was created, dichotomised as 'not in the labour force' and employed. Income was used as it has also been shown to be consistently associated with poorer dietary outcomes among youth ${ }^{(8,30)}$; however, to the best of our knowledge, it has not been tested as a moderator of dietary predictors in children of this age group. Annual gross household income, including pensions and government assistance, was reported using seven income brackets ranging from (1) up to $\mathrm{AU} \$ 12000$ to (7) more than AU\$100000.

\section{Data analysis}

All analyses were conducted separately for boys and girls as preliminary bivariate correlations indicated that there were sex-related differences in unadjusted predictors of dietary intake. First, correlated component regression (CCR) was used (XLSTAT 2012; Addinsoft) to identify the predictors of the three dietary outcomes. CCR employs cross-validation with a step-down algorithm, reducing the number of predictors in the final model by partitioning sample data using bootstrapping into smaller subsets used in multiple regression rounds ${ }^{(31)}$. This regression approach is suited to application within a social-ecological framework as it allows for many independent variables that may be intercorrelated to be 
Table 1. Outcome and predictor variable scales measured in the Child Nutrition Questionnaire (CNQ) and computer-assisted telephone interview (CATI) $†$ (Cronbach's $\alpha$, intraclass correlations (ICC), mean values and standard deviations)

\begin{tabular}{|c|c|c|c|c|c|c|c|c|c|c|c|c|}
\hline \multirow[b]{2}{*}{ Scales } & \multirow[b]{2}{*}{ Survey } & \multirow[b]{2}{*}{ Items } & \multirow[b]{2}{*}{ Response scoring $\ddagger$} & \multirow[b]{2}{*}{ Range } & \multirow[b]{2}{*}{ Cronbach's $\alpha$} & \multirow[b]{2}{*}{ ICC§ } & \multicolumn{2}{|c|}{ All $(n$ 395) } & \multicolumn{2}{|c|}{ Boys $(n 184)$} & \multicolumn{2}{|c|}{ Girls ( $n$ 211) } \\
\hline & & & & & & & Mean & SD & Mean & SD & Mean & SD \\
\hline \multicolumn{13}{|l|}{ Dietary intake scores $\|$} \\
\hline Fruit intake & $\mathrm{CNQ}$ & 4 & $\begin{array}{l}\text { Tick if consumed yesterday; weighted 5-point scale scored from (0) } \\
\text { don't eat fruit to (6) more than five serves per d; number of } \\
\text { fruit consumed from the list }\end{array}$ & $0-16$ & - & - & 5.5 & $2 \cdot 8$ & 5.7 & 3.0 & 5.4 & 2.7 \\
\hline Vegetable intake & $\mathrm{CNQ}$ & 3 & $\begin{array}{l}\text { Tick if consumed yesterday; weighted 5-point scale scored from (0) } \\
\text { don't eat vegetables to }(6) \text { more than five serves per d; number } \\
\text { of vegetables consumed from the list }\end{array}$ & $0-13$ & - & - & 4.7 & $2 \cdot 5$ & 4.6 & $2 \cdot 5$ & 4.8 & 2.5 \\
\hline Healthy behaviours & $\mathrm{CNQ}$ & 5 & $\begin{array}{l}\text { 5-point frequency scale: (1) never/rarely; (2) less than once/week; } \\
\text { (3) 1-3 times/week; (4) 4-6 times/week; (5) every day }\end{array}$ & $4-20$ & 0.54 & - & 17.9 & 3.8 & $17 \cdot 4^{*}$ & 3.7 & $18 \cdot 3^{*}$ & 3.8 \\
\hline \multicolumn{13}{|c|}{ 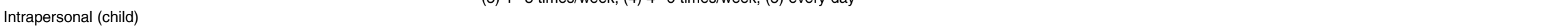 } \\
\hline Self-efficacy for healthy eating & $\mathrm{CNQ}$ & 6 & 5-point agreement scale: $1=\mathrm{SD} ; 2=\mathrm{D} ; 3=\mathrm{NS} ; 4=\mathrm{A} ; 5=\mathrm{SA}$ & $6-30$ & 0.83 & - & 24.2 & $4 \cdot 2$ & 24.3 & 4.4 & $24 \cdot 2$ & 4.0 \\
\hline Attitude to fruit|| & $\mathrm{CNQ}$ & 5 & 5-point agreement scale: $1=\mathrm{SD} ; 2=\mathrm{D} ; 3=\mathrm{NS} ; 4=\mathrm{A} ; 5=\mathrm{SA}$ & $5-25$ & 0.81 & - & 21.8 & 3.1 & 21.9 & 3.2 & 21.6 & $3 \cdot 1$ \\
\hline Attitude to vegetables\| & $\mathrm{CNQ}$ & 4 & 5-point agreement scale: $1=\mathrm{SD} ; 2=\mathrm{D} ; 3=\mathrm{NS} ; 4=\mathrm{A} ; 5=\mathrm{SA}$ & $4-20$ & 0.75 & - & $15 \cdot 3$ & $3 \cdot 1$ & $15 \cdot 2$ & $3 \cdot 1$ & 15.4 & 3.0 \\
\hline Cooking skills & CATI & 2 & 4-point agreement scale: $1=\mathrm{SD} ; 2=\mathrm{D} ; 3=\mathrm{A} ; 4=\mathrm{SA}$ & $2-8$ & 0.62 & $0.88^{* \star}$ & 5.8 & $1 \cdot 1$ & 5.7 & $1 \cdot 1$ & 6.0 & 1.1 \\
\hline \multicolumn{13}{|c|}{ 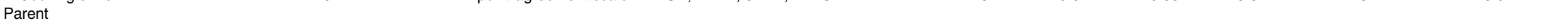 } \\
\hline Parent's fruit and vegetable intake & CATI & 2 & Report number of serves/d & - & - & $0.90^{* *}$ & 4.5 & 1.7 & 4.6 & 1.8 & 4.5 & 1.7 \\
\hline Parent's nutrition knowledge & CATI & 8 & Correct $=1$; incorrect or don't know $=0$ & $0-8$ & 0.62 & $0.49^{\star \star}$ & $7 \cdot 2$ & $1 \cdot 2$ & 7.2 & 1.2 & $7 \cdot 2$ & 1.3 \\
\hline Parent's cooking skills & CATI & 2 & 4-point agreement scale: $1=\mathrm{SD}, 2=\mathrm{D}, 3=\mathrm{A}, 4=\mathrm{SA}$ & $2-8$ & 0.71 & $0.77^{\star *}$ & $6 \cdot 4$ & 1.2 & 6.4 & 1.3 & 6.3 & $1 \cdot 2$ \\
\hline Parent's health considerations & CATI & 6 & 4-point agreement scale: $1=\mathrm{SD}, 2=\mathrm{D}, 3=\mathrm{A}, 4=\mathrm{SA}$ & $4-24$ & 0.87 & $0.61^{\star *}$ & 21.5 & 2.4 & 21.5 & 2.4 & 21.5 & 2.4 \\
\hline Parent's self-efficacy for healthy eating & CATI & 4 & 4 -point scale: $1=\mathrm{No}, 2=\mathrm{SI}, 3=\mathrm{So}, 4=\mathrm{Ve}$ & $4-16$ & 0.70 & $0.73^{\star *}$ & 14.0 & $2 \cdot 0$ & 14.2 & $2 \cdot 0$ & 13.9 & $2 \cdot 0$ \\
\hline Parent's barriers to healthy eatingt† & CATI & 5 & 4-point scale: $1=\mathrm{No}, 2=\mathrm{SI}, 3=\mathrm{So}, 4=\mathrm{Ve}$ & $5-20$ & 0.63 & $0.71^{\star \star}$ & $6 \cdot 8$ & $2 \cdot 1$ & 6.7 & $2 \cdot 3$ & $6 \cdot 8$ & 1.9 \\
\hline Authoritative parenting style $\ddagger \ddagger$ & $\mathrm{CNQ}$ & 6 & 4-point scale: $1=\mathrm{No}, 2=\mathrm{Sl}, 3=\mathrm{So}, 4=\mathrm{Ve}$ & $6-24$ & 0.69 & - & 19.3 & 3.3 & 19.3 & 3.4 & 19.4 & 3.1 \\
\hline Non-authoritative parenting styleł & $\mathrm{CNQ}$ & 3 & 4-point scale: $1=\mathrm{No}, 2=\mathrm{Sl}, 3=\mathrm{So}, 4=\mathrm{Ve}$ & $3-12$ & 0.74 & - & 8.8 & 2.6 & 8.5 & $2 \cdot 7$ & 9.2 & $2 \cdot 4$ \\
\hline Restriction§§ & CATI & 6 & 5-point agreement scale: $1=\mathrm{D}, 2=\mathrm{SI} \mathrm{D}, 3=\mathrm{Ne}, 4=\mathrm{SI} \mathrm{A}, 5=\mathrm{A}$ & $6-30$ & 0.81 & - & $21 \cdot 0$ & 7.4 & 20.7 & 7.6 & $21 \cdot 2$ & $7 \cdot 2$ \\
\hline Food as reward§§ & CATI & 2 & 5-point agreement scale: $1=\mathrm{D}, 2=\mathrm{SI} \mathrm{D}, 3=\mathrm{Ne}, 4=\mathrm{SI} \mathrm{A}, 5=\mathrm{A}$ & $2-10$ & 0.69 & - & 3.7 & 2.5 & 3.5 & $2 \cdot 3$ & 3.9 & $2 \cdot 6$ \\
\hline Pressure to eat§§§ & CATI & 4 & 5 -point agreement scale: $1=\mathrm{D}, 2=\mathrm{SI}, 3=\mathrm{Ne}, 4=\mathrm{SI} \mathrm{A}, 5=\mathrm{A}$ & $4-20$ & 0.64 & - & 9.5 & 4.6 & 9.3 & $4 \cdot 3$ & 9.6 & 4.9 \\
\hline Monitoring food intake§§ & CATI & 4 & 5-point frequency scale: $1=\mathrm{N}, 2=\mathrm{R}, 3=\mathrm{S}, 4=\mathrm{M}, 5=\mathrm{Al}$ & $4-20$ & 0.90 & - & $15 \cdot 9$ & 4.0 & 16.0 & 4.2 & 15.8 & 3.8 \\
\hline Perceived feeding responsibility§§ & CATI & 3 & 5-point frequency scale $1=\mathrm{N}, 2=\mathrm{R}, 3=\mathrm{S}, 4=\mathrm{M}, 5=\mathrm{Al}$ & $3-15$ & 0.68 & - & $12 \cdot 9$ & 1.8 & $13 \cdot 0$ & 1.8 & $12 \cdot 8$ & 1.9 \\
\hline \multicolumn{13}{|l|}{ Home environment } \\
\hline Family barriers to healthy eating & CATI & 4 & $\begin{array}{l}\text { 4-point agreement scale: } 1=\mathrm{SD}, 2=\mathrm{D}, 3=\mathrm{A} \\
\quad 4=\mathrm{SA} ; 4 \text {-point scale: } \\
1=\text { No, } 2=\mathrm{SI}, 3=\text { So, } 4=\mathrm{Ve}\end{array}$ & $4-16$ & 0.74 & $0.55^{\star}$ & 7.8 & $2 \cdot 8$ & 6.7 & $2 \cdot 3$ & 7.7 & $2 \cdot 7$ \\
\hline $\begin{array}{l}\text { Supportive home environment } \\
\text { for healthy eating }\end{array}$ & $\mathrm{CNQ}$ & 7 & 5-point agreement scale: $1=\mathrm{SD}, 2=\mathrm{D}, 3=\mathrm{NS}, 4=\mathrm{A}, 5=\mathrm{SA}$ & $7-35$ & 0.71 & - & $30 \cdot 4$ & 3.4 & 30.7 & 3.5 & $30 \cdot 2$ & $3 \cdot 3$ \\
\hline $\begin{array}{l}\text { Unsupportive home environment for } \\
\text { healthy eating }\end{array}$ & $\mathrm{CNQ}$ & 4 & 5-point agreement scale: $1=\mathrm{SD}, 2=\mathrm{D}, 3=\mathrm{NS}, 4=\mathrm{A}, 5=\mathrm{SA}$ & $4-20$ & 0.69 & - & $10 \cdot 8$ & 3.5 & $10 \cdot 8$ & 3.7 & $10 \cdot 8$ & $3 \cdot 3$ \\
\hline Home fruit and vegetable availability & CATI & 5 & 5-point frequency scale: $1=\mathrm{N}, 2=\mathrm{R}, 3=\mathrm{S}, 4=\mathrm{O}, 5=\mathrm{Al}$ & $5-25$ & 0.52 & $0.62^{\star *}$ & 24.1 & 1.4 & 24.1 & $1 \cdot 2$ & $24 \cdot 1$ & 1.5 \\
\hline Home non-core food availability & CATI & 4 & 5-point frequency scale: $1=\mathrm{N}, 2=\mathrm{R}, 3=\mathrm{S}, 4=\mathrm{O}, 5=\mathrm{Al}$ & $4-20$ & 0.61 & $0.83^{* *}$ & $12 \cdot 8$ & $2 \cdot 9$ & $12 \cdot 8$ & 3.0 & $12 \cdot 7$ & $2 \cdot 8$ \\
\hline \multicolumn{13}{|l|}{ Social environment } \\
\hline Parent upbringing in relation to food & CATI & 2 & 4-point agreement scale: $1=\mathrm{SD}, 2=\mathrm{D}, 3=\mathrm{A}, 4=\mathrm{SA}$ & $2-8$ & 0.75 & $0.91^{\star *}$ & 5.9 & 1.4 & 6.1 & 1.2 & 5.7 & 1.5 \\
\hline Peer influences & $\mathrm{CNQ}$ & 4 & 5-point agreement scale: $1=\mathrm{SD}, 2=\mathrm{D}, 3=\mathrm{NS}, 4=\mathrm{A}, 5=\mathrm{SA}$ & $4-20$ & 0.85 & - & 13.9 & 3.5 & 13.4 & $3 \cdot \overline{6}$ & $14 \cdot 3$ & 3.3 \\
\hline \multicolumn{13}{|l|}{ Neighbourhood environment } \\
\hline Neighbourhood food environment & CATI & 3 & 4-point agreement scale: $1=\mathrm{SD}, 2=\mathrm{D}, 3=\mathrm{A}, 4=\mathrm{SA}$ & $3-12$ & 0.55 & $0.85^{\star \star}$ & 9.8 & 1.5 & 9.8 & 1.5 & 9.7 & 1.5 \\
\hline Perceived cost of healthy foods & CATI & 3 & 4-point agreement scale: $1=\mathrm{SD}, 2=\mathrm{D}, 3=\mathrm{A}, 4=\mathrm{SA}$ & $3-12$ & 0.69 & $0.83^{* *}$ & 6.6 & 1.9 & 6.5 & 1.9 & 6.7 & 1.8 \\
\hline
\end{tabular}

${ }^{*} P<0.05 ;{ }^{* *} P<0.001$.

† All data were collected in Adelaide, South Australia. The participants' age ranged from 9 to 13 years.

¥Agreement scales: SD, strongly disagree; D, disagree; SI D, slightly disagree; Ne, neutral; NS, not sure; A, agree; SI A, slightly agree; SA, strongly agree. General scales: No, not at all; SI, slightly/a little; So, somewhat/a lot; Ve, very much/just like. Frequency scales: N, never; R, rarely; S, sometimes; M, mostly/most of the time; O, often; Al, always.

§ICC were used to determine the test-retest reliability of CATI scores.

$\|$ Score adapted from Wilson et al.(20). For psychometric properties of dietary intake scores refer to Wilson et al. ${ }^{(20)}$.

II Score adapted from Ball et al.(23).

†† Score adapted from Williams et al. (26).

拉 Score adapted from Jackson et al.

$\S \S$ Score adapted from Birch et al. ${ }^{(24)}$. 
Table 2. Demographic characteristics of the study participants* ( $\beta$-Coefficients and, standard errors and $R^{2}$ values)

\begin{tabular}{|c|c|c|c|}
\hline Demographic characteristics & All $(n$ 395) & Boys ( $n$ 184) & Girls $(n 211)$ \\
\hline \multicolumn{4}{|l|}{ Child's age (years) } \\
\hline Mean & $11 \cdot 3$ & $11 \cdot 3$ & $11 \cdot 3$ \\
\hline SD & 0.9 & 0.9 & 0.9 \\
\hline \multicolumn{4}{|l|}{ Mother's age (years) } \\
\hline Mean & $41 \cdot 7$ & $41 \cdot 8$ & $41 \cdot 6$ \\
\hline SD & $5 \cdot 6$ & $5 \cdot 4$ & $5 \cdot 8$ \\
\hline \multicolumn{4}{|l|}{ Sex of the parent completing the CATI† (\%) } \\
\hline Female & $87 \cdot 1$ & 83.7 & $90 \cdot 0$ \\
\hline Male & $12 \cdot 9$ & $16 \cdot 3$ & $10 \cdot 0$ \\
\hline \multicolumn{4}{|l|}{ Marital status (\%) } \\
\hline Partner & $79 \cdot 2$ & $76 \cdot 6$ & $81 \cdot 0$ \\
\hline No partner & $20 \cdot 8$ & $22 \cdot 8$ & $19 \cdot 0$ \\
\hline \multicolumn{4}{|l|}{ Mother’s education levelł (\%) } \\
\hline Did not complete high school & $22 \cdot 8$ & $20 \cdot 1$ & $24 \cdot 8$ \\
\hline Completed high school & $17 \cdot 9$ & $17 \cdot 9$ & $17 \cdot 5$ \\
\hline Trade or diploma & $25 \cdot 6$ & $22 \cdot 3$ & $28 \cdot 2$ \\
\hline University degree & $21 \cdot 5$ & 23.4 & $19 \cdot 4$ \\
\hline Higher university degree & $12 \cdot 2$ & $14 \cdot 1$ & $10 \cdot 2$ \\
\hline \multicolumn{4}{|l|}{ Gross household income§ (\%) } \\
\hline Low & $34 \cdot 3$ & $32 \cdot 9$ & \\
\hline Middle & $36 \cdot 4$ & $36 \cdot 4$ & \\
\hline High & $29 \cdot 2$ & $26 \cdot 6$ & \\
\hline \multicolumn{4}{|l|}{ Household SEIFA\| (\%) } \\
\hline Low & $32 \cdot 9$ & $32 \cdot 1$ & $33 \cdot 6$ \\
\hline Middle & $33 \cdot 2$ & $34 \cdot 2$ & $32 \cdot 2$ \\
\hline High & 33.9 & 33.7 & $34 \cdot 1$ \\
\hline \multicolumn{4}{|l|}{ Mother's occupation I (\%) } \\
\hline Managers and professionals & 30.9 & $31 \cdot 0$ & $30 \cdot 8$ \\
\hline Technicians and trades & 4.8 & $6 \cdot 0$ & $3 \cdot 8$ \\
\hline Community and personal service & 11.4 & $10 \cdot 3$ & $12 \cdot 3$ \\
\hline Clerical, administrative and sales & $22 \cdot 0$ & $25 \cdot 0$ & $19 \cdot 4$ \\
\hline Machinery operators, drivers and labourers & $5 \cdot 6$ & $7 \cdot 6$ & $3 \cdot 8$ \\
\hline Not in the labour force & $20 \cdot 0$ & $15 \cdot 8$ & 23.7 \\
\hline \multicolumn{4}{|l|}{ Country of birth $(\%)$} \\
\hline Australia & $71 \cdot 6$ & $71 \cdot 7$ & 71.6 \\
\hline UK and Ireland & $12 \cdot 7$ & $9 \cdot 8$ & $15 \cdot 2$ \\
\hline Other & $15 \cdot 7$ & 18.5 & $13 \cdot 2$ \\
\hline Aboriginal or Torres Strait Islander (\%) & $3 \cdot 3$ & $3 \cdot 2$ & $3 \cdot 3$ \\
\hline
\end{tabular}

CATI, computer-assisted telephone interview; SEIFA, Socio-Economic Index for Areas.

${ }^{*}$ All data were collected in Adelaide, South Australia. The participants' age ranged from 9 to 13 years.

†Unless otherwise indicated, variables are reported for the parent completing the CATI.

‡Education level was measured on an 8-point scale ranging from 'never attended school' to 'higher university degree'. For ease of reporting, categories 1 to 4 were combined.

$\S$ Gross household income is all household income before tax, including wages, salaries, pensions and allowances, and reported in Australian dollars per annum. Low income $<$ AU $\$ 60000 /$ annum, middle income $=$ AU $\$ 60$ 001-100 000/annum and high income $>$ AU $\$ 100$ 000/annum. Missing income responses $(n 22)$ were categorised as 'refused to answer' or 'unsure'.

\|SEIFA is an area-level measure of socio-economic status determined from home postcode.

I Mother's occupation coded into categories according to the Australian and New Zealand Standard Classification of Occupations. An additional category created for 'not in the labour force' included individuals engaged in full-time home duties, retired persons, unemployed and students. Missing occupation responses ( $n$ 16) were those when participants provided insufficient information for accurate occupation coding.

considered simultaneously ${ }^{(31)}$. All predictor variables were entered into the CCR model along with socio-economic moderators, neighbourhood SEP, child age, marital status and mother's age to account for potential covariates.

To test for moderation, partial least-squares structural equation models were built for each dietary outcome with corresponding predictors, testing for moderation by mother's education, occupation, employment and household income. Models were conducted with resampling by jackknifing using Warp3 PLS (version 2.0; ScriptWarp Systems ${ }^{\mathrm{TM}}$ ). Only variables identified by CCR analysis to predict the corresponding dietary outcome were tested for moderation.
Any covariates identified as predictors (i.e. age and marital status) were not tested for moderation. Additionally, vegetable attitudes was identified as a predictor of fruit intake, but was not tested for moderation as the bivariate correlation of fruit and vegetable attitudes ( $r$ 0.59) indicated that this association may have been due to multicollinearity. This step-wise procedure was employed to reduce the risk of type I errors that would arise from conducting multiple moderation analyses with a large number of predictor variables. To interpret significant moderation effects, predictor variable scales were rescored into tertiles of participant responses, and means of the dietary variable within each tertile of the predictor 
score were graphed. Based on the magnitude of effect sizes determined in other studies assessing the interaction effects of SEP on the predictors of children's dietary intake ${ }^{(10,15,19)}$, in the present study, effect sizes were interpreted as weak if $r<0 \cdot 1$, moderate if $r 0 \cdot 1-0 \cdot 3$ and large if $r>0 \cdot 3$.

\section{Results \\ Descriptive information}

Descriptive statistics are presented in Table 2. Participants were spread relatively evenly across socio-economic strata, except for occupation where fewer mothers were employed as technicians, tradespeople or labourers compared with other occupation types. Most parents who completed the CATI were female $(87 \cdot 1 \%)$, indicating that in this sample, mothers were mostly responsible for their children's food provision. There were no significant differences between boys' and girls' fruit and vegetable intake; however, healthy behaviours were significantly higher among girls (Table 1).

\section{Predictors of healthy eating}

Predictors of fruit and vegetable intake and healthy behaviours were similar for both boys and girls (Table 3). A total of four predictors explained $17 \cdot 0 \%$ of the variance in boys' fruit intake and $11.9 \%$ of the variance in girls' fruit intake: child's self-efficacy for healthy eating, attitudes to fruit and vegetables, and supportive home environment for healthy eating. Attitudes to vegetables and supportive home environment predicted both boys' and girls' vegetable intake, and self-efficacy also predicted girls' vegetable intake. Supportive family environment and attitudes to vegetables explained $12.0 \%$ of the variance in boys' healthy behaviours and supportive family environment and self-efficacy explained $9.4 \%$ of the variance in girls' healthy behaviours. In all instances, a positive relationship was observed whereby more positive attitudes, self-efficacy and supportive home environments predicted higher fruit and vegetable intakes, and more healthy behaviours.

\section{Moderation of healthy dietary intake}

Mothers' education did not act as a moderating variable, and no predictors of girls' fruit and vegetable intake were moderated by SEP (Table 4). Income moderated the associations of boys' fruit and vegetable intake with attitudes. Attitudes were more strongly associated with dietary intake among boys from high-income families (Fig. 1), but the overall gradients were not sharply different, indicating that this moderation

Table 3. Predictors of healthy dietary intake identified using correlated component regression for girls and boys $(n$ 395)*

\begin{tabular}{|c|c|c|c|c|}
\hline \multirow[b]{2}{*}{ Variables } & \multirow[b]{2}{*}{$\mathrm{CV}$ predictor count $\dagger$} & \multicolumn{2}{|c|}{$\begin{array}{l}\text { Regression } \\
\text { coefficients }\end{array}$} & \multirow[b]{2}{*}{ Model goodness-of-fit indices } \\
\hline & & $B$ & $\beta$ & \\
\hline \multicolumn{5}{|l|}{ Girls } \\
\hline \multicolumn{5}{|l|}{ Predictors of fruit intake $(n 211)$} \\
\hline Child's attitude to fruit & 100 & 0.110 & 0.127 & $R^{2} 0.140$ \\
\hline Child's attitude to vegetables & 100 & 0.118 & 0.136 & $R^{2}(\mathrm{CV}) 0.121$ \\
\hline Child's self-efficacy & 99 & 0.071 & 0.107 & SD(CV) 0.005 \\
\hline Supportive family environment & 91 & 0.085 & 0.098 & \\
\hline \multicolumn{5}{|c|}{ Predictors of vegetable intake $(n 211)$} \\
\hline Child's attitude to vegetables & 100 & 0.191 & 0.231 & $R^{2} 0.223$ \\
\hline Supportive family environment & 100 & 0.142 & 0.174 & $R^{2}(\mathrm{CV}) 0.203$ \\
\hline Child's self-efficacy & 100 & 0.107 & 0.170 & $\operatorname{SD}(C V) 0.007$ \\
\hline \multicolumn{5}{|l|}{ Predictors of healthy behaviours ( $n$ 211) } \\
\hline Supportive family environment & 100 & 0.264 & 0.215 & $R^{2} 0.116$ \\
\hline Child's self-efficacy & 90 & 0.171 & 0.181 & $\begin{array}{l}R^{2}(\mathrm{CV}) 0.094 \\
\mathrm{SD}(\mathrm{CV}) 0.008\end{array}$ \\
\hline \multicolumn{5}{|l|}{ Boys } \\
\hline \multicolumn{5}{|l|}{ Predictors of fruit intake ( $n$ 184) } \\
\hline Child's attitude to fruit & 80 & 0.150 & 0.160 & $R^{2} 0.198$ \\
\hline Supportive family environment & 80 & 0.134 & 0.142 & $R^{2}(\mathrm{CV}) 0.172$ \\
\hline Child's self-efficacy & 79 & 0.094 & 0.136 & $S D(C V) 0.005$ \\
\hline Child's attitude to vegetables & 73 & 0.124 & 0.129 & \\
\hline \multicolumn{5}{|l|}{ Predictors of vegetable intake ( $n$ 184) } \\
\hline Child's attitude to vegetables & 80 & 0.341 & 0.306 & $R^{2} 0.235$ \\
\hline Supportive family environment & 80 & 0.294 & 0.269 & $\begin{array}{l}R^{2}(\mathrm{CV}) 0.211 \\
\operatorname{SD}(\mathrm{CV}) 0.010\end{array}$ \\
\hline \multicolumn{5}{|c|}{ Predictors of healthy behaviours ( $n 184)$} \\
\hline Supportive family environment & 80 & 0.291 & 0.250 & $R^{2} 0.148$ \\
\hline Child's attitude to vegetables & 79 & 0.244 & 0.205 & $\begin{array}{l}R^{2}(C V) 0.120 \\
S D(C V) 0.009\end{array}$ \\
\hline
\end{tabular}

$\mathrm{CV}$, cross-validated; $R^{2}(\mathrm{CV})$, variance explained by the final optimised predictive model; SD (CV), standard deviation of $R^{2}$ (CV).

${ }^{*}$ All data were collected in Adelaide, South Australia. The participants' age ranged from 9 to 13 years.

$\dagger$ It represents the number of occasions a variable appeared as a predictor in rounds of cross-validated regression models. For instance, a value of 100 indicates that a predictor appeared in every round of the analyses, whereas a smaller value indicates that a predictor appeared less frequently. It provides an indication of the 'magnitude' of the association in addition to the $\beta$-coefficient. 
Table 4. Moderation of the predictors of healthy dietary intake by socio-economic position using partial least-squares structural equation modelling $(n$ 395)†

( $\beta$-Coefficients, standard errors and $R^{2}$ values)

\begin{tabular}{|c|c|c|c|c|c|c|c|c|c|c|c|c|}
\hline \multirow[b]{2}{*}{ Predictors tested for moderation } & \multicolumn{3}{|c|}{ Education } & \multicolumn{3}{|c|}{ Income } & \multicolumn{3}{|c|}{ Occupation } & \multicolumn{3}{|c|}{ Employment } \\
\hline & $\beta$ & SE & $R^{2}$ & $\beta$ & $\mathrm{SE}$ & $R^{2}$ & $\beta$ & $\mathrm{SE}$ & $R^{2}$ & $\beta$ & $\mathrm{SE}$ & $R^{2}$ \\
\hline \multicolumn{13}{|l|}{ Girls $(n 211)$} \\
\hline \multicolumn{13}{|l|}{ Fruit intake } \\
\hline Fruit attitude & 0.04 & 0.13 & 0.11 & 0.06 & 0.42 & 0.12 & 0.14 & $0 \cdot 12$ & 0.13 & 0.06 & $0 \cdot 10$ & 0.12 \\
\hline Child's self-efficacy & 0.02 & 0.07 & $0 \cdot 10$ & -0.01 & 0.07 & $0 \cdot 10$ & -0.01 & 0.08 & 0.11 & 0.02 & 0.09 & $0 \cdot 10$ \\
\hline Supportive family environment & 0.01 & 0.03 & 0.07 & -0.05 & 0.16 & 0.08 & -0.00 & 0.07 & 0.09 & 0.01 & 0.05 & 0.09 \\
\hline \multicolumn{13}{|l|}{ Vegetable intake } \\
\hline Vegetable attitude & 0.02 & 0.14 & 0.22 & 0.02 & 0.07 & 0.22 & -0.03 & 0.15 & $0 \cdot 23$ & -0.03 & 0.07 & 0.22 \\
\hline Child's self-efficacy & 0.01 & 0.08 & 0.14 & 0.03 & 0.39 & $0 \cdot 13$ & 0.02 & 0.18 & 0.14 & -0.04 & 0.08 & 0.14 \\
\hline Supportive family environment & -0.07 & 0.07 & 0.14 & -0.06 & 0.09 & 0.12 & 0.02 & $0 \cdot 10$ & $0 \cdot 15$ & -0.01 & 0.11 & 0.16 \\
\hline \multicolumn{13}{|l|}{ Healthy behaviours } \\
\hline Child's self-efficacy & -0.03 & $0 \cdot 10$ & 0.12 & 0.06 & 0.83 & 0.08 & $0.09^{*}$ & $0.04^{\star \star}$ & $0.08^{\star \star}$ & 0.08 & 0.07 & 0.08 \\
\hline Supportive family environment & 0.03 & 0.05 & 0.15 & -0.00 & 0.07 & $0 \cdot 10$ & -0.00 & 0.05 & 0.10 & 0.01 & 0.05 & 0.12 \\
\hline \multicolumn{13}{|l|}{ Boys ( $n$ 184) } \\
\hline \multicolumn{13}{|l|}{ Fruit intake } \\
\hline Fruit attitude & 0.02 & 0.05 & 0.17 & $0.07^{\star}$ & $0.05^{\star \star}$ & $0 \cdot 19^{\star \star}$ & -0.09 & $0 \cdot 17$ & 0.21 & 0.01 & 0.09 & $0 \cdot 16$ \\
\hline Child's self-efficacy & 0.06 & 0.07 & 0.17 & 0.04 & 0.09 & $0 \cdot 18$ & -0.09 & 0.09 & $0 \cdot 12$ & 0.09 & 0.14 & 0.15 \\
\hline Supportive family environment & -0.08 & 0.07 & 0.14 & -0.06 & 0.09 & $0 \cdot 16$ & $0.12^{*}$ & $0.05^{\star *}$ & $0 \cdot 17^{\star *}$ & $0.11^{*}$ & $0.06^{\star *}$ & $0.14^{\star *}$ \\
\hline \multicolumn{13}{|l|}{ Vegetable intake } \\
\hline Vegetable attitude & $0 \cdot 10$ & 0.10 & 0.27 & $0 \cdot 11^{*}$ & $0.06^{\star \star}$ & $0.25^{\star \star}$ & -0.07 & 0.07 & 0.25 & 0.01 & 0.09 & 0.24 \\
\hline $\begin{array}{l}\text { Supportive family environment } \\
\text { Healthy behaviours }\end{array}$ & 0.04 & 0.06 & 0.17 & -0.05 & $0 \cdot 10$ & $0 \cdot 18$ & 0.03 & 0.09 & $0 \cdot 16$ & 0.02 & 0.25 & $0 \cdot 16$ \\
\hline Vegetable attitudes & 0.06 & 0.08 & 0.06 & 0.12 & 0.13 & 0.11 & $-0 \cdot 17^{\star}$ & $0.08^{\star *}$ & $0.17^{\star *}$ & -0.06 & 0.08 & $0 \cdot 10$ \\
\hline Supportive family environment & 0.07 & 0.09 & 0.10 & $0.15^{\star}$ & $0.07^{\star \star}$ & $0 \cdot 12^{\star *}$ & -0.06 & 0.08 & 0.19 & -0.01 & 0.10 & 0.15 \\
\hline
\end{tabular}

$\beta$, Path coefficient for interaction term; SE, standard error of path coefficient for interaction term; $R^{2}$, variance explained by the interaction model.

${ }^{*} P \leq 0.05,{ }^{* *} P \leq 0.01$.

† All data were collected in Adelaide, South Australia. The participants' age ranged from 9 to 13 years.

effect was not strong. The association between supportive family environment and boys' fruit intake was moderated by mother's occupation and employment. The moderation effect appeared to be due to a stronger association between boys' fruit intake and supportive family environment for the 'not in the labour force' group compared with the other occupation groups, with no effect in the blue-collar group (Fig. 2). This was confirmed by comparing employed mothers with those 'not in the labour force', which indicated that the moderation effect occurred between employed and unemployed mothers.

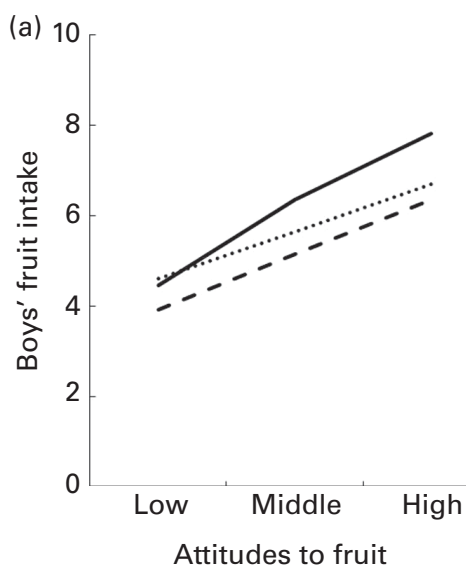

The associations of self-efficacy with girls' healthy behaviours were moderated by mother's occupation, whereby the moderation effect appeared to be due to the differences between the blue-collar workers and the other occupation groups (Fig. 3). Girls of mothers who were blue-collar workers had lower overall healthy behaviours and selfefficacy was unrelated to healthy behaviours. However, this moderation effect was difficult to fully evaluate as no participants in this group reported scores in the high tertile of self-efficacy. In girls of white-collar workers, professionals and mothers 'not in the labour force', higher self-efficacy for

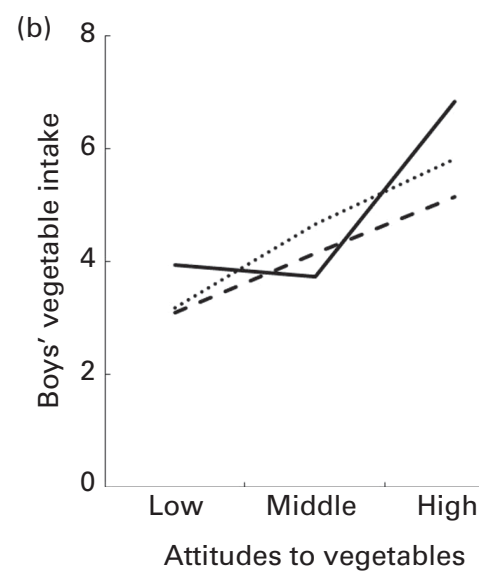

Fig. 1. Moderation effects of income on the associations of attitudes with boys' (a) fruit and (b) vegetable intake. Moderation effects were significant at $P \leq 0.05$. (a) Attitudes to fruit tertiles of participant scores (low $=6-21$; middle $=22-23$; high $=24-25$ ). (b) Attitudes to vegetable tertiles of participant scores (low = 4-14; middle = 15-17; high = 18-20). ....... Low income; - -, middle income; _ _, high income. 


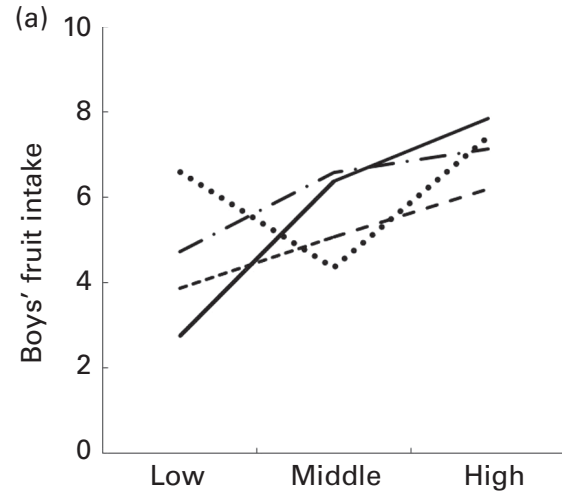

Supportive family environment

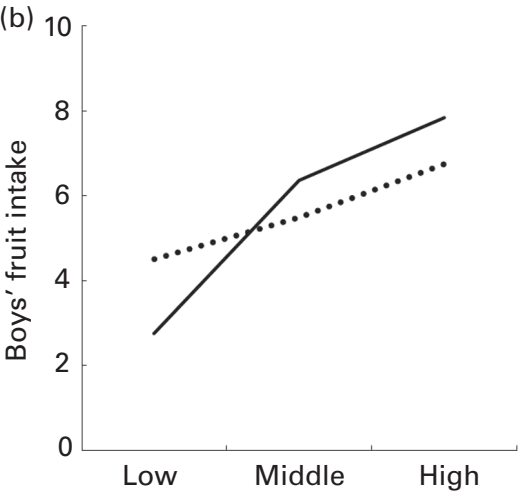

Supportive family environment

Fig. 2. Moderation effects of occupation and employment on the associations of supportive family environments with boys' fruit intake. Moderation effects were significant at $P \leq 0.05$. (a, b) Supportive family environment tertiles of participant scores (low $=7-24$; middle $=25-27$; high $=28-35$ ). (a) ......, Blue-collar workers; ----, white-collar workers; — ., professionals; —, 'not in labour force' group. (b) ......, Employed; —, 'not in labour force' group.

healthy eating was related to higher healthy behaviour scores. This relationship appeared to be strongest for girls of mothers 'not in the labour force'. For boys, the associations of healthy behaviours with vegetable attitudes and supportive family environment were moderated by income, employment and occupation (Fig. 3). The association between supportive family environment and boys' healthy behaviours was strongest in the high- and middle-income groups. The effect of attitudes on boys' healthy behaviours was somewhat equivocal for mothers 'not in the labour force' and blue-collar workers. In contrast, there was a strong, positive, linear relationship for the professional group, whereby more positive attitudes were associated with higher healthy behaviours. A similar but weaker association of attitudes with healthy behaviours occurred for the white-collar group.

\section{Discussion}

The present study used a social-ecological framework to consider the role of personal, home, social and neighbourhood factors on children's dietary intake, and whether these factors interacted with SEP. A combination of personal and home environment factors predicted children's healthy dietary intake, and SEP moderated a number of the identified predictor-dietary intake relationships. The findings of the present study, alongside the small number of earlier studies ${ }^{(9,10,15,19)}$ suggest that predictors of food intake differ between children of low and high SEP, and therefore it may be of benefit to tailor dietary interventions for different socio-economic groups. A considerable number of interventions targeting dietary change, obesity reduction or prevention have had only limited success that may not be sustained in the long term $^{(32,33)}$. One of the reasons may be that the impact of interventions is contingent on population-specific factors such as the sex or SEP of participants, and for interventions to be successful, the socio-economic context of participants should be considered ${ }^{(34)}$. Most dietary interventions in children are directed at a diverse range of participants from across the socio-economic spectrum ${ }^{(33,35)}$. The focus of children's dietary interventions has been predominantly nutrition education, as
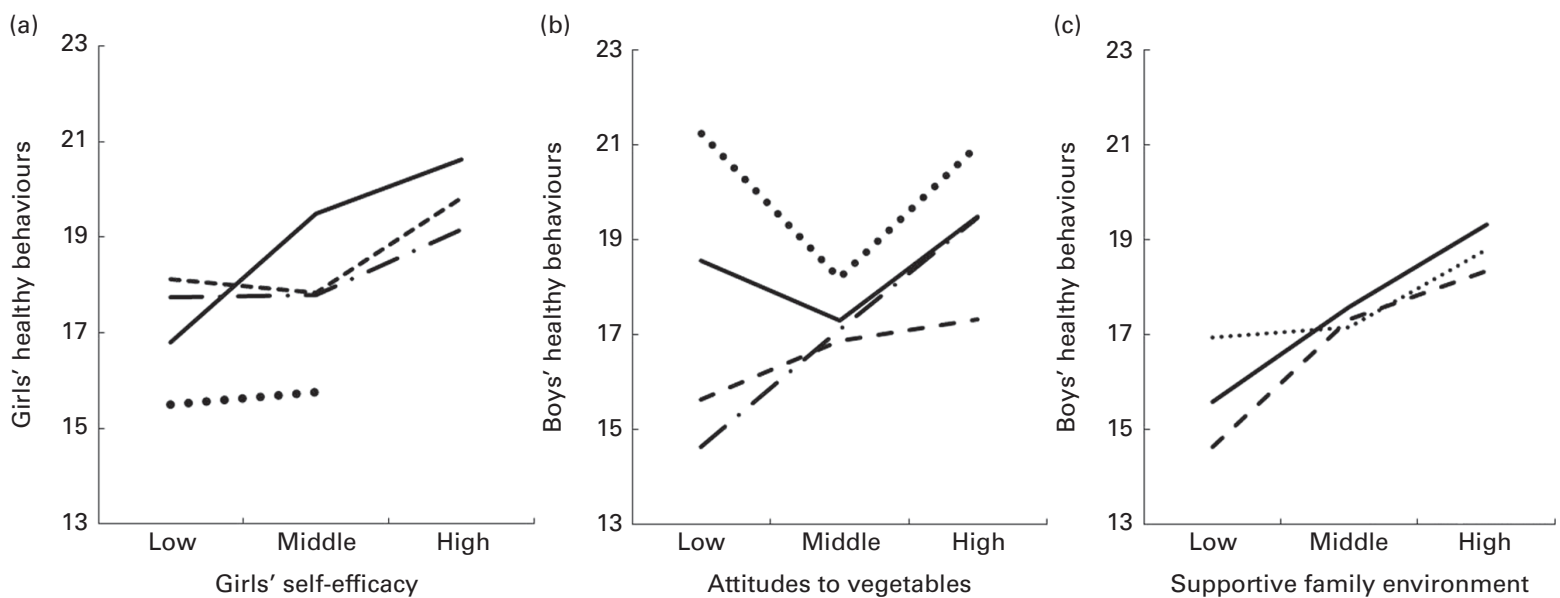

Fig. 3. Moderation effects of occupation and income on the associations of correlates with children's healthy behaviours. Moderation effects were significant at $P \leq 0.05$. (a) Girls' self-efficacy tertiles of participant scores (low $=6-22$; middle $=23-26$; high $=27-30$ ). (b) Attitudes to vegetable tertiles of participant scores (low $=4-14$; middle $=15-17$; high $=18-20)$. (c) Supportive family environment tertiles of participant scores (low $=7-24$; middle $=25-27$; high $=28-35)$. (a, b) ....., Blue-collar workers; -.--, white-collar workers; - ., professionals; —, 'not in labour force' group. (c) ......., Low income; - - , middle income; _ , high income. 
well as changing food availability in settings such as schools and community stores ${ }^{(32,33,36)}$. The present study suggests that parent-focused interventions may be of benefit. Moderation results indicated that it may be beneficial to instruct low-SEP parents about creating home environments that are supportive of healthy eating, by making fruits and vegetables more accessible, and modelling healthy behaviours themselves. Consistent with social-ecological theories of healthy behaviours, moderation findings highlighted the importance of developing cognitive traits, including self-efficacy and attitudes, for healthy eating. However, helping parents to create supportive home environments for healthy eating may in itself support children to develop more positive attitudes, knowledge and self-efficacy for healthy eating.

Healthy dietary intake was predicted by a combination of positive attitudes, self-efficacy and a supportive family environment. Cognitive factors, in particular self-efficacy and attitudes, have been recognised as important for understanding SEP variations in healthy dietary intake in youth ${ }^{(7)}$. The present study found that a supportive family environment was the most consistent predictor of all healthy dietary intake outcomes. In accordance with social-ecological models, both cognitive and environmental factors are likely to be necessary for healthy eating, as cognitive factors are important in driving decisions to make healthy food choices, but it is not possible for a child to act on these decisions without an environment that supports healthy eating ${ }^{(22,37)}$. The most proximal environment influencing children's eating behaviour is the home. The home environment, and in particular availability of and access to fruits and vegetables, has been consistently identified as a correlate of children's fruit and vegetable intake ${ }^{(4)}$. Interestingly, child-reported perceptions of the home food environment in the supportive environment scale, and none of the parentreported home environment scales, predicted healthy food intake. This suggests that children's perceptions of the home environment are particularly important influences on healthy food intake in this age group. Children learn from their experiences with food in their home, thereby forming attitudes and self-efficacy for healthy eating, and a combination of these factors is likely to contribute to healthy eating, as was observed in the present study.

Consistent with other studies, self-efficacy was positively associated with fruit and vegetable intake and healthy behaviours $^{(38,39)}$. Self-efficacy represents a belief in one's ability to overcome barriers to healthy eating ${ }^{(40)}$. In environments where fruit and vegetable availability may be low, for instance in low-SEP families, self-efficacy may be an important enabler for fruit and vegetable consumption ${ }^{(41)}$. Sandvik et al. ${ }^{(15)}$ found that occupation moderated the effects of self-efficacy on intention to eat fruit, with the strongest positive relationship of self-efficacy and intention to eat fruit in the low-SEP group. In the present study, associations of self-efficacy with healthy behaviours were also moderated by occupation, but the opposite relationship to that found by Sandvik et al. ${ }^{(15)}$ was observed, with self-efficacy unrelated to healthy behaviours in the low-SEP group. However, when considering only the low tertile of supportive family environment scores, children of low-income families reported higher mean healthy behaviour scores than those from the middle- and highincome groups (Fig. 3). This may indicate that other factors such as cognitive variables (i.e. self-efficacy), influenced healthy behaviours of children from low-income families in home environments with lower support for healthy eating, as proposed by Kratt et al. ${ }^{(41)}$. Although there appear to be different relationships between self-efficacy and healthy eating in different SEP groups, few studies have measured this relationship, and self-efficacy has been measured in different ways across studies, so the nature of this relationship is unclear and warrants further investigation.

The associations of boys' attitudes to fruit and vegetables with intake of fruit and vegetables were also moderated by income, with stronger associations being observed among high-income parents. Similar to the findings of Sandvik et al. ${ }^{(15)}$ for stronger associations of self-efficacy with fruit intake in low-SEP groups, it was expected that attitudes would be more strongly associated with fruit and vegetable intake in low-SEP families, where attitude scores may expect to be more variable. A stronger association in the higher-SEP group suggests greater variability in attitudes, which is a stronger limiting factor in this group. It may be that children's attitudes to healthy eating are influenced by home environment factors that themselves are variable, including parent's nutrition knowledge, parent modelling and home food availability ${ }^{(41)}$. Parents of high SEP may themselves perceive health and nutrition to be more important, and may therefore be more likely to encourage children to consume healthy foods, thereby influencing their attitudes to fruits and vegetables ${ }^{(15,42,43)}$. It is likely that these factors have a complex relationship, and the interactions between child cognitive factors and family environment factors (including SEP) in determining fruit and vegetable intake fit within social-ecological frameworks for understanding children's dietary behaviours ${ }^{(22)}$.

The present study found no association of supportive home environments with healthy eating in low-SEP families, and a positive association between supportive home environment and boys' healthy behaviours in high-income families. This is consistent with emerging evidence of socio-economically related differences in home environment factors supporting healthy eating. Fruit and vegetable availability may be poorer in low-SEP households, compared with high-SEP households ${ }^{(5,7,8,44)}$, and has been shown to be related to fruit intake in high-SEP groups but not in low-SEP groups, suggesting that in low-SEP households fruit may not be sufficiently available to influence intake ${ }^{(15)}$. In a study involving 12- to 15-year-old children, perceived fruit and vegetable accessibility explained $50-90 \%$ of socio-economic disparities in fruit and vegetable intake ${ }^{(8)}$. An Australian study involving 12- to 15-year-old adolescents of low SEP found that those who were always served vegetables at dinner were at least twice as likely to consume vegetables frequently than those who were served vegetables with dinner less frequently ${ }^{(45)}$. There may also be a significant difference in parent facilitation, for instance cutting up fruit for children, between low- and high-SEP groups ${ }^{(15)}$, and parents of high SEP may eat more fruits and vegetables themselves, thereby modelling more positive eating behaviours to their children ${ }^{(7,8,19,43)}$. 
All data were self-reported and therefore subject to misreporting or socially desirable response bias, which may have affected responses non-randomly across the SEP groups. There are limitations associated with children's ability to accurately recall and report their dietary intake; however, the CNQ has acceptable validity and reliability ${ }^{(20)}$, and children of this age are capable of self-reporting dietary intake $^{(46)}$. It must be recognised that causal pathways cannot be fully determined from the present cross-sectional study. The strengths of the present study were that the predictors of boys' and girls' dietary intake were evaluated separately, and a theoretically driven step-wise statistical approach was employed. The CCR allowed for the comparison of multiple indicators from a social-ecological framework to identify predictors of each dietary outcome before testing for moderation of relevant predictor variables by SEP indicators. The survey instruments were validated with good psychometric properties. Children's questionnaires were administered online to reduce missing data and errors associated with data entry. The moderation effects of four SEP indices were evaluated to provide a better understanding of the role SEP plays in modifying the determinants of children's dietary intake. Finally, the participant sample was recruited from diverse regions of metropolitan Adelaide, distributed relatively evenly across the SEP indicators evaluated.

\section{Conclusions}

The present study found that children's healthy dietary intake was predicted by attitudes, self-efficacy and supportive family environments for healthy eating. The moderation effects of SEP variables were small overall, although some moderation effects that were identified across multiple SEP indicators may be proposed as targets of health promotion and interventions to improve dietary intake of different SEP groups. In particular, these findings suggest that helping parents to create supportive home environments from which children may develop more positive attitudes and self-efficacy for healthy eating may contribute to improvements in dietary intake among children of low SEP.

\section{Supplementary material}

To view supplementary material for this article, please visit http://dx.doi.org/10.1017/S0007114514001354

\section{Acknowledgements}

The authors acknowledge the contribution of Keren Kneebone who coordinated the study and conducted the data collection. The authors also acknowledge the schools, children and parents who gave their time to participate in the present study. John Petkov, a much loved and respected statistician, sadly passed away in January 2013. The authors are very grateful for his contribution to the present study and for his generous mentorship over the years.

The present study was supported by an Australian Research Council (ARC) Linkage Grant with SA Health (no. LP 0883949).
N. P. was supported by the National Health and Medical Research Council Program Grant funding (no. 320860 and 631947). The ARC and SA Health had no role in the design, analysis or writing of this article.

The authors' contributions are as follows: D. M. Z., N. P. and J. D. contributed to the study design, statistical analysis and interpretation of the data; D. M. Z. carried out the data collection and drafted the manuscript; N. P. and J. D. revised the manuscript; D. M. Z. conceptualised and conducted the statistical analysis, with the assistance of a statistician. All authors read and approved the final manuscript.

The authors have no conflicts of interest to declare.

\section{References}

1. Cameron AJ, Ball K, Pearson N, et al. (2012) Socioeconomic variation in diet and activity-related behaviours of Australian children and adolescents aged 2-16 years. Pediatr Obes 7, 329-342.

2. Shrewsbury V \& Wardle J (2008) Socioeconomic status and adiposity in childhood: a systematic review of cross-sectional studies 1990-2005. Obesity (Silver Spring) 16, 275-284.

3. Stalsberg R \& Pedersen AV (2010) Effects of socioeconomic status on the physical activity in adolescents: a systematic review of the evidence. Scand J Med Sci Sports 20, 368-383.

4. Rasmussen M, Krølner R, Klepp K, et al. (2006) Determinants of fruit and vegetable consumption among children and adolescents: a review of the literature. Part I: quantitative studies. Int J Behav Nutr Phys Act 3, 22.

5. Hilsen M, van Stralen MM, Klepp K-I, et al. (2011) Changes in 10-12 year old's fruit and vegetable intake in Norway from 2001 to 2008 in relation to gender and socioeconomic status - a comparison of two cross-sectional groups. Int J Behav Nutr Phys Act 8, 108.

6. Department of Health and Ageing (2008) National Australian Children's Nutrition and Physical Activity Survey: Main Findings. Canberra: Commonwealth Government of Australia.

7. Ball K, MacFarlane A, Crawford D, et al. (2009) Can social cognitive theory constructs explain socio-economic variations in adolescent eating behaviours? A mediation analysis. Health Educ Res 24, 496-506.

8. Bere E, van Lenthe F, Klepp K-I, et al. (2008) Why do parents' education level and income affect the amount of fruits and vegetables adolescents eat? Eur J Public Health 18, 611-615.

9. Ho S-Y, Wong BY-M, Lo W-S, et al. (2010) Neighbourhood food environment and dietary intakes in adolescents: sex and perceived family affluence as moderators. Int J Pediatr Obes 5, 420-427.

10. Wouters EJ, Larsen JK, Kremers SP, et al. (2010) Peer influence on snacking behavior in adolescence. Appetite 55, 11-17.

11. Neumark-Sztainer D, Wall M, Perry C, et al. (2003) Correlates of fruit and vegetable intake among adolescents. Findings from Project EAT. Prev Med 37, 198-208.

12. Moore GF, Tapper K, Murphy S, et al. (2007) Associations between deprivation, attitudes towards eating breakfast and breakfast eating behaviours in 9-11-year-olds. Public Health Nutr 10, 582-589.

13. O'Dea JA \& Wilson R (2006) Socio-cognitive and nutritional factors associated with body mass index in children and adolescents: possibilities for childhood obesity prevention. Health Educ Res 21, 796-805. 
14. Edmonds J, Baranowski T, Baranowski J, et al. (2001) Ecological and socioeconomic correlates of fruit, juice and vegetable consumption among African-American boys. Prev Med 32, 476-481.

15. Sandvik C, Gjestad R, Samdal O, et al. (2010) Does socio-economic status moderate the associations between psychosocial predictors and fruit intake in schoolchildren? The Pro Children study. Health Educ Res 25, 121-134.

16. Fairchild AJ \& MacKinnon DP (2009) A general model for testing mediation and moderation effects. Prev Sci 10, 87-99.

17. Frazier PA, Tix AP \& Barron KE (2004) Testing moderator and mediator effects in counseling psychology research. J Couns Psychol 51, 115-134.

18. Lien N, Jacobs DR \& Klepp K-I (2002) Exploring predictors of eating behaviour among adolescents by gender and socio-economic status. Public Health Nutr 5, 671-681.

19. Rodenburg G, Oenema A, Kremers SPJ, et al. (2012) Parental and child fruit consumption in the context of general parenting, parental education and ethnic background. Appetite 58, $364-372$

20. Wilson A, Magarey A \& Mastersson N (2008) Reliability and relative validity of a child nutrition questionnaire to simultaneously assess dietary patterns associated with positive energy balance and food behaviours, attitudes, knowledge and environments associated with healthy eating. Int $J$ Behav Nutr Phys Act 5, 5-17.

21. Bronfenbrenner U (1979) The Ecology of Human Development: Experiments by Nature and Design. Cambridge, MA: Harvard University Press.

22. Sallis JF, Owen N \& Fisher EB (2008) Ecological models of health behavior. In Health Behavior and Health Education: Theory, Research and Practice, pp. 465-485 [K Glanz, BK Rimer and K Viswanath, editors]. San Francisco, CA: Jossey-Bass.

23. Ball K, Crawford D \& Mishra G (2006) Socioeconomic inequalities in women's fruit and vegetable intakes: a multilevel study. Public Health Nutr 9, 623-630.

24. Birch LL, Fisher JO, Grimm-Thomas K, et al. (2001) Confirmatory factor analysis of the Child Feeding Questionnaire: a measure of parental attitudes, beliefs and practices about child feeding and obesity proneness. Appetite 36, 201-210.

25. Jackson C, Bee-Gates D \& Henriksen L (1994) Authoritative parenting, child competencies, and initiation of cigarette smoking. Health Educ Q 21, 103-116.

26. Williams L, Ball K \& Crawford D (2010) Why do some socioeconomically disadvantaged women eat better than others? An investigation of the personal, social and environmental correlates of fruit and vegetable consumption. Appetite 55, 441-446.

27. Pallant J (2010) SPSS Survival Manual: A Step by Step Guide to Data Analysis Using SPSS, 4th ed. Maidenhead: Open University Press.

28. Hannon P, Bowen D, Moinpour C, et al. (2003) Correlations in perceived food use between the family food preparer and their spouses and children. Appetite 40, 77-83.

29. Australian Bureau of Statistics (2006) Australian and New Zealand Standard Classification of Occupations (ANZSCO) First Edition (ABS Cat. No. 1220.0). Canberra: ABS.
30. Golley RK, Hendrie GA \& McNaughton SA (2011) Scores on the dietary guideline index for children and adolescents are associated with nutrient intake and socio-economic position but not adiposity. J Nutr 141, 1340-1347.

31. Magidson J, (2010) Correlated Component Regression: A Prediction/Classification Methodology for Possibly Many Features. American Statistical Association Proceedings, Vancouver. Alexandria, VA: American Statistical Association.

32. Campbell K, Campbell K, Waters E, et al. (2001) Interventions for preventing obesity in childhood. A systematic review. Obes Rev 2, 149-157.

33. Waters E, de Silva-Sanigorski A, Hall BJ, et al. (2011) Interventions for preventing obesity in children. The Cochrane Database of Systematic Reviews, issue 12 CD001871.

34. Campbell M, Fitzpatrick R, Haines A, et al. (2000) Framework for design and evaluation of complex interventions to improve health. BMJ 321, 694-696.

35. Brug J, van Lenthe FJ \& Kremers SPJ (2006) Revisiting Kurt Lewin: how to gain insight into environmental correlates of obesogenic behaviors. Am J Prev Med 31, 525-529.

36. Blanchette L \& Brug J (2005) Determinants of fruit and vegetable consumption among 6-12-year-old children and effective interventions to increase consumption. J Hum Nutr Diet 18, 431-443.

37. Stokols D (1992) Establishing and maintaining healthy environments. Toward a social ecology of health promotion. Am Psychol 47, 6-22.

38. De Bourdeaudhuij I, te Velde S, Brug J, et al. (2008) Personal, social and environmental predictors of daily fruit and vegetable intake in 11-year-old children in nine European countries. Eur J Clin Nutr 62, 834-841.

39. Haerens L, Craeynest M, Deforche B, et al. (2008) The contribution of psychosocial and home environmental factors in explaining eating behaviours in adolescents. Eur J Clin Nutr 62, 51-59.

40. Bandura A (1997) Self-efficacy: The Exercise of Control. New York, NY: W.H. Freeman and Company.

41. Kratt P, Reynolds K \& Shewchuk R (2000) The role of availability as a moderator of family fruit and vegetable consumption. Health Educ Behav 27, 471-482.

42. Hupkens CLH, Knibbe RA \& Drop MJ (2000) Social class differences in food consumption: the explanatory value of permissiveness and health and cost considerations. Eur $J$ Public Health 10, 108-113.

43. Rimal RN (2003) Intergenerational transmission of health: the role of intrapersonal, interpersonal, and communicative factors. Health Educ Behav 30, 10-28.

44. MacFarlane A, Crawford D, Ball K, et al. (2007) Adolescent home food environments and socioeconomic position. Asia Pac J Clin Nutr 16, 748-756.

45. Stephens LDA, McNaughton SA, Crawford D, et al. (2011) Correlates of dietary resilience amongst socioeconomically disadvantaged adolescents. Eur J Clin Nutr $\mathbf{6 5}$, 1219-1232.

46. Baranowski T \& Domel SB (1994) A cognitive model of children's reporting of food intake. Am J Clin Nutr 59, $212 S-217 S$ 